Campos Neutrais - Revista Latino-Americana de Relações Internacionais

Vol. 1 No 1, Janeiro - Abril de 2019

\title{
La dinámica de seguridad entre los grandes poderes y sus efectos en las relaciones en el ámbito de la defensa entre el hegemón y su región: El caso de los Estados Unidos y América Latina
}

Resumen: ¿Qué factores explican las relaciones en el ámbito de la defensa entre los Estados Unidos de América (EUA) y los Estados de América Latina (AL)?Desde el surgimiento de la Doctrina Monroe en el siglo XIX hasta el la caída del muro de Berlín en 1989, los EUA han tenido como objetivo principal el mantener su influencia en la región y consolidar los intereses de seguridad norteamericanos en ella, manteniendo al resto de los grandes poderes competidores fuera de América. Con el fin de la Guerra Fría, la unipolaridad y la consecuente ausencia de imperativos de seguridad permitió un creciente ascenso de la agenda económica, dejando los temas de defensa en un segundo plano.En este sentido, el trabajo se sostiene que el poder junto con la competencia por el mismo entre las grandes potencias del sistema internacional son variables fundamentales para analizar las relaciones en el ámbito de la defensa entre los EUA y los Estados de AL, ya que la voluntad de los EUA de dominar el continente americano sumado a la presencia de desafíos por parte de potencias extrarregionales en los distintos momentos de la historia ayuda a entender y explicar la dinámica que guió desde principios del siglo XIX hasta la actualidad el relacionamiento entre los EUA y AL en el campo de la defensa y la seguridad.

Palabras-clave: Defensa. América Latina. Distribución internacional del poder.

Abstract: What factors explain the relations in the field of defense between the United States of America and the Latin American States? Since the emergence of the Monroe Doctrine in the nineteenth century until the fall of the Berlin Wall in 1989, the United States has had as its main objective to maintain its influence in the region and consolidate US security interests in it, keeping the rest of the great powers competing outside of America. With the end of the Cold War, unipolarity and the consequent absence of security imperatives allowed a growing rise of the economic agenda, leaving defense issues in the background. In this sense, the work argues that

Licencido en Ciencia Política por la Universidad de Buenos Aires. Maestrando en Estudios Internacionales en la Universidad Torcuato Di Tella. 
Campos Neutrais - Revista Latino-Americana de Relações Internacionais

Vol. 1 No 1, Janeiro - Abril de 2019

power and the competition for it among the great powers of the international system are fundamental variables to analyze the relations in the field of defense between the US and the LA States, since the will of the USA to dominate the American continent, together with the presence of challenges from outside the region in the different moments of history helps to understand and explain the dynamics that led from the beginning of the XIX century to the present day the relationship between the USA and $\mathrm{AL}$ in the field of defense and security.

Keywords: Defence. Latin America. International distribution of power.

\section{Introducción}

¿Qué factores explican las relaciones en el ámbito de la defensa entre los Estados Unidos de América (EUA) y los Estados de América Latina (AL)? El presente artículo buscará dar respuesta a esta pregunta.

$\mathrm{Si}$ uno tiene en consideración la conducta de los EUA frente a los Estados latinoamericanos en el campo de la seguridad, puede observarse que la misma siempre osciló de acuerdo a poder estadounidense y a la distribución internacional de poder. A mediados del siglo XIX, cuando los EUA estaban ascendiendo en la jerarquía del sistema internacional, fue el momento fue el momento en el que el país del norte lanzó la Doctrina Monroe, en donde la misma estaba claramente orientada a prevenir la incursión de potencias europeas en el territorio americano, especialmente aquellas zonas -como América Central- que estaban estrechamente ligados con los intereses estadounidenses. Desde esta doctrina hasta el final de la Guerra Fría, puede observarse como los EUA han buscado relacionarse con los Estados del sur a partir de la búsqueda por consolidar su poder en el continente y por mantener a las potencias extrarregionales al márgen. Esto puede reconocerse en poíticas como las del "buen vecino", la del "gran garrote", el Tratado Interamericano de Asistencia Recíproca, la Doctrina de la Seguridad Nacional y el Plan Cóndor, entre otras. Con el fin de la Guerra Fría, la unipolaridad y la consecuente ausencia de imperativos de seguridad debido a la desarticulación de la Unión Soviética, permitió un creciente ascenso de la agenda económica, dejando los temas de defensa en un segundo plano.

En este sentido, el trabajo se sostiene que el poder junto con la competencia por el mismo entre las grandes potencias del sistema internacional son variables fundamentales para analizar las relaciones en el ámbito de la defensa entre los EUA y 
Campos Neutrais - Revista Latino-Americana de Relações Internacionais

Vol. 1 No 1, Janeiro - Abril de 2019

los Estados de AL. En otras palabras, la voluntad de los EUA de dominar el continente americano sumado a la presencia de desafíos por parte de potencias extrarregionales a este objetivo en los distintos momentos de la historia es un factor que no puede dejar de ser tenido en cuenta a la hora buscar entender y explicar la dinámica que guió desde principios del siglo XIX hasta la actualidad el relacionamiento entre los EUA y AL en el campo de la defensa y la seguridad.

En función de lo mencionado, se identificará a la distribución internacional de poder como variable independiente y a las relaciones en el ámbito de la defensa entre los EUA y los Estados de AL como variable dependiente, y se buscará describir cómo las relaciones entre los EUA y $\mathrm{AL}$ en el campo de la defensa fueron oscilando en función de los cambios de la distribución internacional de poder y de la voluntado de los EUA por manterer la región latinoamericana estable y sin interferencias extrarregionales.

\section{Realismo ofensivo y dinámica de seguridad regional entre grandes poderes}

La teoría del realismo estructural ofensivo de Mearsheimer (2014), al hacer referencia a la dinámica de la cometencia de poder entre los grandes poderes del sistema internacional, es harto relevante para entender y explicar las relaciones en el ámbito de la denfensa entre los EUA y los países de AL.

La construcción teórica establece que; en primer lugar, la búsqueda de seguridad (supervivencia) el principal objetivo de todos los Estados; en segundo lugar, que no es posible saber las intenciones de las demás unidades que conforman el sistema internacional; en tercer lugar, que todos los Estados tienen capacidad ofensiva para dañar a otras unidades que conforman el sistema internacional; en cuarto lugar, que los Estados son racionales en el sentido que conocen el escenario en el cual se mueven y se comportan estratégicamente para cumplir sus objetivos y; en quinto lugar, que el sistema internacional es anárquico, es decir, que no hay una autoridad superior para dirimir conflictos y garantizar la seguridad.

A partir de estos axiomas, se concluye que la mejor opción que tienen los Estados para lograr sobrevivir en el sistema internacional es la de maximizarel poder. En este sentido, se desprende de lo mencionado que el objetivo de las unidades del 
Campos Neutrais - Revista Latino-Americana de Relações Internacionais Vol. 1 No $^{\circ}$, Janeiro - Abril de 2019

sistema sea el de convertirse en hegemón ${ }^{1}$, ya que, por su misma definición, implica que el Estado hegemónico es tan poderoso que ningún otro Estado tiene la capacidad militar convencional suficiente para batirse en una guerra con él y salir victorioso. En otras palabras, cuando un Estado logra convertirse en hegemón tiene la supervivencia garantizada y deja de buscar maximizar su poder.

Ahora bien, el ser hegemón también implica llegar a una situación en donde un Estado acumula el poder suficiente para dominar todo el sistema. Sin embargo, el globo es tan grande que resulta muy difícil para un Estado el proyectar poder a lo largo de todo el planeta. Además, el autor introduce el concepto de 'stopping forces' (como los océanos) que contribuyen a hacer realmente muy complicada la tarea de dominar absolutamente todo el sistema internacional.

Este factor geográfico lleva a que en la teoría se realice la distinción entre hegemones globales y hegemones regionales. Los primeros tienen el poder sufuciente para dominar todo el sistema, evitar el surgimiento de poderes revisionistas (es decir, son statu-quoistas) y se caracterizan por lograr el dominio mencionado a través de la superioridad nuclear ${ }^{2}$. Los segundos están caracterizados por dominar una parte del sistema internacional, generalmente la ligada a su región más próxima.

La consideración de hegemones regionales es relevante ya que permite ver cuál es la dinámica de seguridad e influencia entre grandes poderes que están separados por factores geográficos, como por ejemplo los EUA y China. En este sentido, el realismo estructural ofensivo indica que, a pesar de estar separados por 'stopping forces', los hegemones regionales intentar impedir que otros Estados alejados obtengan la hegemonía de su región.

Por un lado, los hegemones regionales prefieren que en las demás regiones del mundo haya al menos dos o más grandes poderes, así dichos Estados están preocupados gastanto energía y recursos compitiendo entre ellos y dejando menos margen a que alguno pueda convertirse en una potencial amenaza. En otras palabras, Mearsheimer indica que si el poder en una región está distribuido de forma relativamente pareja3 3 , el hegemón regional distante puede quedarse tranquilo de que

\footnotetext{
${ }^{1} \mathrm{El}$ autor menciona que en sintonía con este objetivo se encuentran la voluntad de acumular riqueza, tener preeminencia territorial y conseguir la superioridad nuclear.

${ }^{2}$ Por ejemplo, EEUU desde 1945 hasta que la Unión de Repúblicas Socialistas Soviéticas descubrió dicha tecnología. Para un desarrollo más amplio del ejemplo, consultar el capítulo 12 del libro $L a$ Diplomacia de Kissinger.

3 La teoría hace la diferenciación entre distintas distribuciones de poder que implican una mayor/menor estabilidad y, por ende, una mayor/menor amenaza para la seguridad de los Estados. Ver el capítulo 8 del libro 'The Tragedy of Great Power Politics' de Mearsheimer.
} 
Campos Neutrais - Revista Latino-Americana de Relações Internacionais Vol. 1 No $^{\circ}$, Janeiro - Abril de 2019

su seguridad no será amenazada en el corto/mediano plazo por otro hegemón regional. De todas formas, frente al surgimiento de otro Estado que domine su región, el Estado lejano que ya había logrado dominar su propia región y que buscaba impedir el surgimiento del primero puede realizar acciones orientadas a balancear el poder de éste.

Por otro lado, cuando ya hay dos hegemones regionales consolidados, Mearsheimer (2014) menciona que pueden amenazarse el uno al otro ayudando a romper el balance de poder favorable al hegemón en su región. Por ejemplo, Nixon buscando romper el balance de poder a favor de la Unión Soviética mediante su alianza con China a principios de 1970, lo que implicó que el Estado soviético tenga que preocuparse no solo por su frente occidental sino también por el oriental. Además, un hegemón regional también tiene fuertes incentivos para ayudar a algún Estado de otra región distante que sea revisionista y quiera desafiar al hegemón de su zona. Es decir, cuando los Estados ya lograron dominar su región, éstos van a buscar competir con otros hegemones regionales intentando desestabilizar su 'patio trasero' y alterar4 el balance de poder a favor de los Estados 'challangers' que cuestionen y revisen el statu-quo regional.

De esta estrategia se deduce que los Estados que tienen o aspiran a lograr la hegemonía regional buscan dominar, consolidar y mantener estable su propia región frente a las embestidas de terceros Estados. Por lo tanto, la dinámica está caracterizada por hegemones regionales que buscan mantener el statu quo en su región mientras tratan de desestabilizar y alterar el balance de poder en regiones lejanas dominadas por hegemones regionales rivales.

Con respecto al concepto de "defensa", tomaremos la definición que brinda Eissa basándose en Battaglino, en donde menciona que la politica de defensa puede ser definida como "el conjunto de acciones que adopta un Estado para garantizar su supervivencia frente a riesgos y amenazas" (EISSA, 2013, p. 174). En este sentido, y considerando el marco teórico expuesto, podemos mencionar que cuando hablamos de "relaciones que tienen los Estados en el ámbito de la defensa" estamos considerando todos aquellos intercambios materiales, discursivos y simbólicos que

\footnotetext{
$4 \mathrm{El}$ realismo estructural ofensivo establece distintas estrategias mediante las cuales los grandes poderes buscan maximizar su poder en detrimento del poder de los demás. Pueden (1) balancear, (2) hacer un 'back-passing', (3) ir a la guerra, (4) usar la herramienta del chantaje y, finalmente, utilizar la estrategia (5) 'bait and bleed' o (6) ‘bloodletting'. Ver capítulo 5 del libro 'The Tragedy of Great Power Politics' de Mearsheimer.
} 
Campos Neutrais - Revista Latino-Americana de Relações Internacionais

Vol. 1 No 1, Janeiro - Abril de 2019

llevan adelante dos o más Estados con el objetivo de buscar proteger a sus respectivas ciudadanías de amenazas y riesgos de origen externo.

Ahora bien, si tenemos en cuenta que los EUA fueron ascendiendo y consolidándose como hegemón regional desde finales del siglo XIX, podemos analizar las relaciones que ha mantenido con los Estados latinoamericanos en el ámbito de la defensa a partir de 3 factores: (1) la distribución internacional del poder, (2) de sus intentos por colocarse como hegemón regional a medida que acumulaba poder entre finales del siglo XIX hasta la Segunda Guerra Mundial y (3) por sus efuerzos realizados para mantener la estabilidad y garantizar el statu quo en su región una vez consolidado como el Estado más poderoso del continente americano desde el final de la Segunda Guerra Mundial hasta la actualidad.

\section{Principios del siglo XIX. Multipolaridad y emergencia de los EUA como poder regional}

El 2 de diciembre de 1823 el presidente estadounidense James Monroe dio su mensaje anual en el Congreso y, en dicho mensaje, estableció tres puntos que iban a ser nodales para la política exterior norteamericana. En primer lugar, que los EUA no se involucrarían en una guerra librada en el continente europeo. En segundo lugar, estableció que su país no toleraría que los poderes europeos adquieran territorio del continente americano para incrementar su poder. En tercer lugar, que los EUA querían asegurarse que ningún país europeo establezca alianzas con con Estados independientes del hemisferio occidental ni los domine de ninguna manera.5 En relación a estas declaraciones del presidente estadounidense, puede verse como el Estado del norte comenzaba -aún teniendo un poder reativo similar o menor al de las potencias europeas- a intentar consolidar su presencia en el continente americano, en donde un paso fundamental para la consecución de esto era el impedir que una potencia extracontinental desestabilice la zona mediante la conquista de territorio o a través la formación de alianzas. De esta forma, si bien los EUA "no tenía suficiente capacidad militar para hacer cumplir la Doctrina Monroe en las primeras décadas en la que esta fue enunciada” (MEARSHEIMER, 2014, p. 249), el Estado del norte comenzaba mediante una acción discursiva a delinear sus objetivos en el campo de la defensa para con el resto del continente americano, en donde la presencia o

\footnotetext{
5 Ver capítulo 7 del libro ‘The Tragedy of Great PowerPolitics'de Mearsheimer (2014).
} 
Campos Neutrais - Revista Latino-Americana de Relações Internacionais

Vol. 1 No 1, Janeiro - Abril de 2019

intervenciones de potencias europeas en su región se veían como un riesgo al interés estadounidense.

Para finales del siglo XIX, en 1889 se realizó la Primera Conferencia Panamericana en donde el gobierno estadounidense propuso una iniciativa panamericana que tenía como objetivo "crear una unión aduanera que facilitara el comercio entre las naciones del hemisferio y dejara a los europeos en posición de inferioridad"(ESCUDÉ y CISNEROS, 2000, p. Tomo IX) ${ }^{6}$. Si bien esta propuesta tenía una finalidad comercial y no prosperó en gran medida debido a la intransigencia de los negociadores argentinos Saenz Peña y Quintana, es interesante ver la continuidad de la postura estadounidense en términos de política exterior para con su región. La voluntad del presidente Monroe en 1823 de mantener a las potencias extracontinentales fuera de su incipiente zona de influencia seguía muy firme en 1889.

\section{Siglo XX: Multipolaridad y búsqueda de los EUA de establecer su dominio en la $\mathrm{AL}$}

En esta misma línea y ya con un EUA que igualaba a las potencias europeas en términos de poder relativo, durante la presidencia (1901 a 1909) de Theodore Roosevelt se proclamó el "Corolario Roosevelt a la Doctrina Monroe"7, en donde los EUA se guardaba el derecho a intervenir ${ }^{8}$ de forma preventiva en los asuntos de otras naciones americanas en caso de que estas estén realizando acciones que puedan ser consideradas lesivas para el interes estadounidense en la región (definido a partir de la Doctrina Monroe). A partir de este momento, "la seguridad hemisférica sería la piedra angular del papel mundial de Estados Unidos, basado en la decidida afirmación del interés nacional". Es decir, el Estado del norte buscaba mantener el orden, la estabilidad y seguridad en su continente para poder consolidar la base que luego le permita actuar en el resto de los asuntos internacionales.

En este sentido, la administración de T. Roosevelt se caracterizó por la política del 'Big Stick' en su relación en el ámbito de la defensa con AL. Esta política puede reflejarse en la voluntad del gobierno estadounidense de intervenir en los asuntos

\footnotetext{
${ }^{6}$ Link presente en la bibliografía.

7 Ver capítulo 7 del libro Orden Mundial de Kissinger.

8 Se destacan las intervenciones a Haití, Nicaragua, República Dominicana y en la revolución mexicana.
} 
Campos Neutrais - Revista Latino-Americana de Relações Internacionais

Vol. 1 No $^{\circ}$, Janeiro - Abril de 2019

domésticos de los Estados latinoamericanos (principalmente centroamericanos9) en caso que estos lleven adelante acciones contrarias al interés nacional estadounidense. Un ejemplo claro de la política del 'Gran Garrote' es la imposición de la Enmienda Platt a la Constitución Cubana en 1901 luego de la expulsión de España de la isla. En el punto III de dicha Enmienda se menciona que:

Estados Unidos puedan ejercitar el derecho de intervenir para la conservación de la Independencia cubana, el mantenimiento de un Gobierno adecuado para la protección de vidas, propiedad y libertad individual y para cumplir las obligaciones que con respecto a Cuba han sido impuestas a los Estados Unidos [...] (ENMIENDA PLATT, 1901, s/p).

Otro hecho relevante durante la presidencia de T. Roosevelt por los importantes efectos que tuvo para asegurar la seguridad hemisférica deseada por los EUA fue la creación del canal de Panamá10, ya que el mismo le permitió a la armada estadounidense navegar entre los Océanos Atlántico y Pacífico sin tener que realizar la circunnavegación por Cabo de Hornos.

Ya a partir de 1933, el gobierno de Franklin Delano Roosevelt afrontó un escenario caracterizado por los problemas económicos producto de la gran depresión de 1929 y por una distribución de poder favorable a su país, ya que Europa estaba sofocada por problemas económicos y por las consecuencias materiales, económicas y sociales que produjo la entonces llamada "Gran Guerra". En este contexto, en donde ya era difícil pensar que habían amenazas y riesgos de intervenciones europeas en el continente americano, el proyecto que F.D Roosevelt llevó adelante para consolidar la seguridad hemisférica fue la denominada "Política del Buen Vecino".

Esta política comenzó en la VII Conferencia Panamericana realizada en Montevideo en 1933. En el encuentro los Estados latinoamericanos lograron la adopción de la Convención sobre Derechos y Deberes de los Estados, en donde“El artículo principal de la Convención era el que disponía que 'ningún Estado tiene derecho de intervención en los asuntos internos ni en los externos de otro"' (ESCUDÉ

9 Como los EUA recién estaban empezando a despegarse de las potencias europeas respecto a su poder relativo y a consolidar su zona de influencia en la región, la voluntad de intervenir en los asuntos domésticos se la relaciona principalmente con los Estados de Centroamérica, ya que eran los que podían dañar más al país del norte con sus comportamientos díscolos debido a su cercanía con este país.

${ }^{10}$ La creación de dicho canal estuvo caracterizada por la fuerte influencia estadounidense para lograr la independencia de Panamá, ya que el territorio en donde se construyó el canal era originalmente de Colombia. 
Campos Neutrais - Revista Latino-Americana de Relações Internacionais Vol. 1 No 1, Janeiro - Abril de 2019

y CISNEROS, 2000, p. Tomo IX) ${ }^{11}$. El concepto de 'intervención' era considerado estrictamente como 'intervención militar' y fue un cambio sustantivo en lo que hizo a las relaciones de EEUU y AL en el ámbito de la defensa, ya que se dejó atrás las implicancias imperialistas de la Doctrina Monroe y su Corolario para comenzar a rechazar las intervenciones unilaterales y empezar a pensar en la seguridad hemisférica a partir del mínimo acuerdo con el resto de los Estados latinoamericanos.

En términos concretos, la 'política del buen vecino' implicó el retiro de tropas de Haití y reconocimiento a la Independencia de este país junto con la Derogación de la Enmienda Platt. A su vez, contribuyó a que la respuesta estadounidense a la nacionalización de petroleras mexicanas no sea a través de una intervención militar sino a partir de la exigencia de indemnizaciones.

\section{Fin de la Segunda Guerra Mundial e inicio de la Guerra Fría. Bipolaridad} y búsqueda de los EUA por consolidar su dominio en la AL y mantener alejada a la Unión Soviética

Con la finalización de la Segunda Guerra Mundial, la distribución internacional de poder pasó a estar caracterizada por la bipolaridad del sistema internacional. Por un lado, los EUA y, por el otro, la Unión de Repúblicas Socialistas Soviéticas. En este sentido, los EUA buscaron orientar las relaciones en el ámbito de la defensa con los Estados latinoamericanos a partir del Tratado Interamericano de Asistencia Recíproca (TIAR) ${ }^{12}$ firmado 1947 y regulado mediante el Consejo Permanente de la Organización de los Estados Americanos (OEA) que fue fundada en 1948.

El TIAR es considerado una alianza de seguridad colectiva en la medida que "la ideología de los Estados que, particularmente dispuestos a atacar, pretenden establecer un principio de que un ataque contra ellos debe convertirse en una razón de preocupación para otros Estados" (CARR en COMINI, 2014, p. 15-16). Es decir, los esquemas de seguridad colectiva tienen lógica cuando surgen en contextos donde hay un alto grado de incertidumbre o cuando un grupo de Estados identifica de forma clara a otros Estados que pueden proporcionarles una amenaza, como sucedió con los EUA y la URSS durante la Guerra Fría. En este sentido, lo expuesto sobre el TIAR se observa en su artículo 3.1, en donde menciona que:

\footnotetext{
${ }^{11}$ Link de la página web presente en la bibliografía.
}

1223 estados fueron signatarios del tratado. 
Las Altas Partes Contratantes convienen en que un ataque armado por parte de cualquier Estado contra un Estado Americano, será considerado como un ataque contra todos los Estados Americanos, y en consecuencia, cada una de dichas Partes Contratantes se compromete a ayudar a hacer frente al ataque, en ejercicio del derecho inmanente de legítima defensa individual o colectiva que reconoce el Artículo 51 de la Carta de las Naciones Unidas (TRATADO INTERAMERICANO DE ASISTENCIA RECÍPROCA, 1947, s/p).

Luego del triunfo de la revolución cubana en 1959 y la ruptura de sus relaciones diplomáticas con Washington, los EUA buscaron expulsar a Cuba de la OEA con el apoyo del resto de los países latinoamericanos y lograr que éstos rompan las relaciones diplomáticas con la isla. El rápido apoyo que la URSS le dio a la revolución cubana hizo que el objetivo que tenían los EUA de mantener estable y dominada su región (es decir, fuera del alcance de la URSS y de la ideología comunista) se vea comprometido, lo que llevó a la Batalla de Playa Girón que consistió en la invasión de Cuba por parte de cubanos exiliados con el apoyo de los EUA.

La influencia de la URSS tan cerca geográficamente de los EUA dio pie para uno de los mayores incidentes de la Guerra Fría: la crisis de los misiles. Esta crisis tuvo lugar en 1962 y consistió en el descubrimiento por parte del gobierno estadounidense de la presencia de bases de vectores nucleares de alcance medio en territorio cubano. La crisis culminó con un bloqueo estadounidense a la isla para evitar que siga el abastecimiento de material bélico nuclear de la isla y el inicio de las negociaciones entre el presidente Kennedy y su contraparte de la URSS, Nikita Jruschov. Las mismas culminaron en el retiro del material nuclear de Cuba por parte de la URSS y el retiro de misiles estadounidenses que estaban ubicados en Turquía.

Estos riesgos y amenazas que sufrieron los EUA y que fueron en gran medida consecuencia de la presencia de la URSS en el continente americano llevó a que el país del norte busque estrechar sus relaciones en el ámbito de la defensa con el resto de los Estados de su región. Este contexto y la presencia de una distribución rígida de poder caracterizada por la bipolaridad influyó en la creación en los EUA de la Doctrina de Seguridad Nacional13 (DSN). La misma fue aplicada a partir de 1960 en

\footnotetext{
${ }_{13}$ "La DSN fue un conjunto de concepciones o cuerpo de enseñanza derivado de supuestas verdades, principios, normas y valores que un Estado, a través de sus propias experiencias o las de otros Estados y de conformidad con su Constitución Política y con las realidades del país, considera que debe llevar a la práctica para garantizar el desarrollo integral del hombre y de la colectividad nacional, preservándolos de interferencias a perturbaciones sustanciales de cualquier origen" (VELÁSQUES, 2002, p. 12).
} 
Campos Neutrais - Revista Latino-Americana de Relações Internacionais

Vol. 1 No 1, Janeiro - Abril de 2019

los Estados latinoamericanos y estaba basada en una interpretación particular de la Guerra Fría, en donde el:

[...] mundo contemporáneo estaba dividido en las dos fracciones irreconciliables indicadas, el occidente cristiano y el oriente comunista. Este enfrentamiento se manifestaba mediante la subversión interna que permitía el desarrollo del conflicto sin el riesgo de una guerra de aniquilamiento suicida (VELÁSQUES, 2002, p. 13).

Esta subversión provenía del bloque comunista y atentaba contra la seguridad misma del Estado, de su ciudadanía y de los valores occidentales que esa Nación representaba. Por ende, para la DNS "la guerra tenía un nuevo sentido : total y global, indivisible y permanente, puesto que todo estaba implicado y la agresión podía venir tanto del interior como del exterior, el comunismo se filtraba por todas partes" (VELÁSQUES, 2002, p. 14). Este nuevo significado que cobró la guerra llevó a la consideración de que las fronteras entre los Estados no servían para ganar una guerra que era total e indivisible, por lo que surgió el concepto de "fronteras ideológicas". En este sentido, las Fuerzas Armadas de los países jugaron un rol fundamental en este nuevo 'escenario de batalla', ya que las mismas estaban a cargo de llevar delante esta guerra total contra la subversión del enemigo interno comunista con el objetivo de proteger la unidad nacional y los valores occidentales.

Bajo la DSN, se da impulso a la cooperación y el entrenamiento de las Fuerzas Armadas de los países latinoamericanos en la Escuela de las Américas, en donde el entrenamiento estaba orientado hacia el perfeccionamiento del combate de contrainsurgencia, ya que esta estrategia era la que llevaba adelante el considerado "enemigo interno".

En sintonía con esto, también se destacó en este escenario el llamado "Plan Cóndor" que tuvo lugar entre fines de los 70' y comienzos de los 80'. Como indica el IPPDH (2015) ${ }^{14}$, el mismo tuvo el aval de los EUA (la creación de este plan le es atribuida a Kissinger, el Secretario de Estado de Nixon) y fue un esquema de coordinación de acciones, apoyo mutuo y logística entre los principales comandantes de las Fuerzas Armadas del cono sur que mantuvo como principal objetivo en términos políticos y de seguridad el desmantelar todas las organizaciones internas de cada Estado miembro que puedan ser consideradas como subversivas bajo los parámetros de la DSN. Es decir, correspondió a la llamada "guerra total" bajo la

14Ver IPPDH: A 40 años del Cóndor (2015). 
Campos Neutrais - Revista Latino-Americana de Relações Internacionais

Vol. 1 No 1, Janeiro - Abril de 2019

lógica de la lucha contra el comunismo, en donde las fronteras ideológicas eran fundamentales para entender la lógica del conflicto.

\section{Fin de la Guerra Fría. Unipolaridad y dominio incontestado de los EUA en AL}

Terminada la Guerra Fría y con el advenimiento de una distribución de poder unipolar marcadamente favorable a los EUA. En este escenario caracterizado por un déficit de amenazas ${ }^{15}$, el Estado del norte dio a sus relaciones con el resto de los países latinoamericanos una impronta marcadamente económica, caracterizada por el surgimiento del Consenso de Washington. A su vez, esto significo una subordinación de los asuntos hemisféricos vinculados al ámbito de la defensa, relajando las presiones que los EUA históricamente ejercieron para con los Estados de AL y permitiendo que estos ganen mayor autonomía ${ }^{16}$ en sus conductas vinculadas a la defensa y la seguridad.

Es en este contexto cuando comienzan a desarrollarse un gran número de instituciones latinoamericanas orientadas a fomentar la cooperación y la coordinación en ámbitos de defensa. En este sentido, desde principios de la década del 90' Suramérica experimentó un creciente proceso de interacción entre los Estados y las elites civiles y militares, en donde se buscó reconceptualizar y repensar ciertos presupuestos establecidos en el campo de la defensa. Este proceso se inició en el Consenso de Santiago firmado en el marco de la Organización de Estados Americanos (OEA) en 1991, en donde se reflejó el "compromiso reflejado en la voluntad regional de avanzar en la eliminación de hipótesis de conflicto, mejorar la cooperación militar, apoyar la estabilidad democrática, fomentar el control civil sobre el sector militar y promover la transparencia de las políticas de defensa" (BATTAGLINO, 2012, p. 89).

Desde la firma del Consenso de Santiago, el camino orientado hacia una mayor coordinación, consulta y transparencia en materia de defensa se continuó y profundizó de manera notable. Battaglino (2012) indica que a partir de 1995 tuvieron lugar nueve Conferencias de Ministros de Defensa en la OEA, sumado al hecho de que aumentaron los mecanismos de cooperación bilateral entre países suramericanos que hacía algunas décadas estaban inmersos en un ambiente de desconfianza por las

\footnotetext{
15Ver Buzan (2006).

${ }^{16}$ Autonomía en términos de Realismo Periférico. Ver el libro Realismo Periférico de Carlos Escudé.
} 
Campos Neutrais - Revista Latino-Americana de Relações Internacionais Vol. 1 No $^{\circ}$, Janeiro - Abril de 2019

múltiples hipótesis de conflicto, como por ejemplo Argentina con Brasil y Chile. Además, desde 1995 muchos países suramericanos comenzaron a publicar su información militar -cuyo conocimiento por otros países hubiese sido considerado hasta hace algunos años como un asunto de amenaza a su seguridad nacional- como medio para reducir la incertidumbre.

Ya en la primera década del siglo XXI, a la unipolaridad favorable a los EUA y a la ausencia de una amenaza hemisférica concreta se le sumaron otros factores que contribuyeron a un cambio en las relaciones en el ámbito de la defensa entre los EUA y los Estados de AL. En primer lugar, el cambio en la estrategia estadounidense ${ }^{17}$ luego del 11 de septiembre del 2001, en donde se llevó adelante la "guerra contra el terror" y la preocupación por las "nuevas amenazas", en donde el principal enemigo estadounidense no eran concretamente algún Estado-Nación, sino que eran organizaciones terroristas que en todo caso podían estar auspiciadas por Estados ${ }^{18}$. En segundo lugar, la reactivación de la IV flota de Estados Unidos para controlar el Caribe y Sudamérica que estaba desactivada desde el fin de la segunda guerra mundial. En tercer lugar, como menciona Battaglino (2012), Brasil en su carácter de líder regional siempre intentó proyectarse y cumplir sus aspiraciones internacionales usando a Suramérica como plataforma. Es por esto que las posibilidades de conflicto interestatal (como la crisis protagonizada entre Colombia y Ecuador en 2008) fueron vistos por Brasil como hechos que tenían el poder de afectar su liderazgo regional y, por ende, su proyección y aspiraciones internacionales.

Este conjunto de factores llevó a que los Estados sudamericanos comiencen a pensar en una seguridad garantizada no por las acciones unilaterales del Estado, sino por la acción coordinada, la transparencia de datos y los mecanismos de consulta entre las distintas partes. Esto llevó a la creación en el marco de la Unasur del Consejo Sudamericano de Defensa (CDS).

Las alianzas de seguridad cooperativa son definidas como "sistemas de interacciones estatales que, coordinando políticas gubernamentales, previenen y contienen las amenazas a los intereses nacionales y evitan que las percepciones que de estas tienen los diversos Estados se transformen en tensiones, crisis o abiertas confrontaciones" (VARAS en COMINI, 2014, p. 15). Por lo tanto, lo que caracteriza a los esquemas de seguridad cooperativa es la búsqueda de prevención de conflictos,

17 Ver la 'National Security Strategy' de los EUA del 2002.

${ }^{18}$ Bush en su momento definió como eje del mal a Irán, Corea del Norte e Iraq. 
Campos Neutrais - Revista Latino-Americana de Relações Internacionais

Vol. 1 No $^{\circ}$, Janeiro - Abril de 2019

crisis y tensiones a partir del fomento de la confianza. Este objetivo puede ser conseguido con mayor facilidad mediante la creación de una institución -como el CDS, por ejemplo- que tenga mecanismos orientados a aumentar la confianza y bajar la incertidumbre. Entre estos mecanismos se incluyen, entre otros, a la coordinación política, la consulta permanente, organismos preparados para la solución de conflictos y la publicación de información de tipo militar.

A su vez, la creación del CDS implicaba dejar a los EUA fuera de una alianza de seguridad en donde participaban todos los países sudamericanos. Como menciona Nolte (2018), esto contribuyó asolapar parcialmente las funciones de la Organización de Estados Americanos en lo que respecta a tareas de seguridad regional, ya que los Estados sudamericanos dejaron de utilizar tantoa la OEA para comenzar a resolver las cuestiones de seguridad regional en el marco y con un mayor protagonismo del CDS, como por ejemplo, en los casos de la crisis de Colombia en 2009, la crisis entre Colombia y Venezuela en 2010, y entre Colombia y Venezuela en 2015.

\section{Palabras finales}

El presente artículo buscó mostrar como las relaciones en el ámbito de la defensa entre los EUA y los Estados de AL estuvieron fuertemente condicionadas por la participación del hegemón del norte en la distribución internacional del poder y por los esfuerzos de este mismo Estado por consolidar la estabilidad y su dominio en la región, lo que a su vez implicaba mantener alejada la posibilidad de cualquier intervención de un gran poder extrarregional

En este sentido, se introdujo al realismo estructural ofensivo como marco teórico para intentar entender la dinámica competitiva que se da entre dos hegemones regionales que están separados por 'stopping forces'. En donde cada uno busca mantener estable y dominar su propia región mientras que, al mismo tiempo, buscan desestabilizar e impedir que el hegemón rival mantenga el control de su área de influencia.

En función de este marco teórico, se hizo un recorrido histórico de las relaciones entre los EUA y AL en el ámbito de la defensa desde la creación de la Doctrina Monroe hasta la primera década del siglo XXI. A partir de este análisis, se sostuvo que estas relaciones siempre estuvieron condicionadas por la distribución internacional de poder y, en esta misma línea, se argumentó que, en los momentos en donde la distribución de poder se caracterizaba por la presencia de un competidor al 
Campos Neutrais - Revista Latino-Americana de Relações Internacionais Vol. 1 No 1, Janeiro - Abril de 2019

poderío estadounidense, el hegemón americano buscó reforzar las relaciones en defensa con los países latinoamericanos.

En suma, este breve recorrido histórico muestra que las relaciones que los EUA y los Estados de AL han tenido en el ámbito de la defensa respondieron, principalmente, a factores estructurales vinculados a las distintas distribuciones internacionales de poder y, secundariamente, a factores coyunturales ligados a la estrategia y objetivos inmediatos de los EUA. Por un lado, cuando los EUA buscaron consolidar su influencia en el continente con una distribución de poder multipolar, las relaciones de defensa entre EUA y AL estuvieron atravesadas por a Doctrina Monroe y su Corolario. Por otro lado, cuando el país del norte buscó mantener la estabilidad en su región en un contexto bipolar, las relaciones entre las partes analizadas se caracterizaron por tener de base al TIAR y, dos décadas más tarde, a la DSN. Por último, cuando el final de la Guerra Fría marcó una distribución de poder unipolar favorable a los EUA, los Estados de AL obtuvieron mayor autonomía para llevar adelante sus relaciones en el ámbito de la defensa y la seguridad, lo que les permitió desde desarticular las hipótesis de conflicto hasta crear el CDS.

\section{Bibliografía}

BATTAGLINO, Jorge. Defence in a post-hegemonic regional agenda: The case of the South American Defence Council. En Pía Riggirozzi \& Diana Tussie (Org.).The rise of post-hegemonic regionalism: The case of Latin America. London: Springer, 2012, p. 81-100.

BUZAN, Barry. Will the 'global war on terrorism' be the new Cold War? International Affairs, v.82, p.1101-1118, 2006.

COMINI, Nicolás. El rol del Consejo de Defensa de la UNASUR en los últimos conflictos regionales.Revista Nueva Sociedad, n 230, p. 14-22, 2010.

EISSA, Sergio. Política exterior y política de defensa en la Argentina: dos caras de la misma moneda. Revista perspectivas de Política Pública, v3. $n^{\circ} 5$, p. 171-191, 2013.

ESCUDE, Carlos\&CISNEROS. Andrés.A Sétima Conferencia Internacional dos Estados Americanos. Disponible en:http://www.argentina-rree.com/9/9oo6.htm. Acceso en 4/10/2018.

ESCUDE, Carlos. Realismo Periférico: Fundamentos para la nueva política exterior argentina, Argentina: Planeta, p. 79-147, 1992.

IPPDH - INSTITUTO DE POLÍTICAS PÚBLICAS EN DERECHOS HUMANOS MERCOSUR.A 4o años del Cóndor: De las coordinaciones represivas a la construcción de las políticas públicas regionales en derechos humanos. 2015. Disponible en:http://www.raadh.mercosur.int/wp-content/uploads/2015/o6/A-40años-del-Cóndor.pdf. Acceso en 14/10/2018. 
Campos Neutrais - Revista Latino-Americana de Relações Internacionais

Vol. 1 No 1, Janeiro - Abril de 2019

KISSINGER, Henry. La Diplomacia. México D.F: Fondo de Cultura Económica, 1994.

KISSINGER, Henry. Orden Mundial. Buenos Aires: Debate, 2014.

MEARSHERIMER, John.The Tragedy of Great Power Politics. New York: W.W. Norton Company Inc, 2014.

NOLTE, Detlef. Costs and Benefits of Overlapping Regional Organizations in Latin America: The Case of the OAS and UNASUR. Latin Aerican Politics and Society, v. 6o, p. 128-153, 2018.

VELASQUES RIVERA, Edgar de Jesús. Historia de la Doctrina de Seguridad Nacional. Convergencia. Revista de Ciencias Sociales, v.9, $\mathrm{n}^{\circ}$ 27, p. 11-39, 2002.

WALTZ, Kenneth.Theory of International Politics. United States of America: Waveland Press Inc, 2010.

\section{Documentos Oficiales}

CONSENSO DE WASHINGTON. 1989. Disponible en:

https://piie.com/publications/papers/williamsono904-2.pdf.

ENMIENTA PLATT. 1935. Disponible en:

http://cael.reduniv.edu.cu/pluginfile.php/1050/mod_folder/content/o/Documentos \%20hist\%C3\%B3ricos\%20del\%2oper\%C3\%ADodo\%201899- 1935 / Alteração\% 2oPlatt.pdf? Forcedownload $=1$.

TRATADO INTERAMERICANO DE ASISTENCIA RECÍPROCA. 1947. Disponible en:http://www.oas.org/juridico/spanish/tratados/b-29.html. 